\title{
An angular spectral method for solution of the heat equation in spheroidal geometries
}

\author{
D. J. Ivers*
}

(Received 28 October 2004, revised 24 June 2005)

\begin{abstract}
A spectral numerical method is presented for solving the heat equation in oblate or prolate spheroids. Cartesian coordinates are scaled to transform the spheroidal geometry into a spherical geometry. The diffusion term in the transformed equation is anisotropic, being enhanced in the polar directions. The transformed equation is discretised in angle using a truncated spherical harmonic expansion of the temperature in transformed spherical polar coordinates. The anisotropic diffusion term is reduced to block tridiagonal form using recurrence relations for spherical harmonics. The radial coordinate is discretised using finite differences in scaled radius but other radial schemes are possible. Without heat sources and with a homogeneous Dirichlet boundary condition the problem reduces to an eigenproblem for the decay rate. The results are compared to the separated
\end{abstract}

${ }^{*}$ School of Mathematics and Statistics, University of Sydney, Sydney, Australia. mailto:D. Ivers@maths. usyd.edu.au

See http://anziamj.austms.org.au/V46/CTAC2004/Iver for this article, (C) Austral. Mathematical Soc. 2005. Published September 1, 2005. ISSN 1446-8735 
variables solution, which employs oblate or prolate spheroidal wave functions. The method directly extends to other scalar problems in spheroidal geometries, which have the highest derivatives in Laplacian form, including passive advection-diffusion of a scalar. The method may be extended, with difficulty, to problems with vector diffusion or ellipsoidal geometries.

\section{Introduction}

Thermal conduction in solid prolate and oblate spheroids is considered. The partial differential equation governing the temperature $\Theta$ is

$$
\partial_{t} \Theta=\kappa \nabla^{2} \Theta+\frac{Q}{\rho c_{p}},
$$

where $\kappa$ is the uniform thermal diffusivity, $Q$ is the heat production per unit volume, $\rho$ is the mass density, and $c_{p}$ is the specific heat capacity at constant pressure. On the boundary the temperature satisfies Dirichlet or Robin conditions;S

$$
\Theta=\Theta_{0} \quad \text { or } \quad \partial_{n} \Theta+\alpha \Theta=\Theta_{0}^{\prime} .
$$

The problem without heat sources was originally solved by Niven [5] either for boundary condition (2) with $\Theta_{0}=0$, or for $\Theta_{0}^{\prime}=0$ and $\alpha$ uniform.

The present study was originally motivated by the close relation between the oblate spheroidal heat conduction problem and the turbulent anisotropic thermal diffusion models for the Earth's core considered by Phillips and Ivers $[6,7,8]$ and Ivers [3]. The convective state of the Earth's core is almost certainly turbulent and beyond the resolution of present numerical geodynamo simulations. In the Braginsky and Meytlis [1] picture of core turbulence, the transport of momentum and heat is enhanced in preferred directions such as the rotation axis. Thus the simplest rapid rotation model 
for the turbulent thermal diffusion tensor is $\mathbf{D}_{\kappa}=\kappa_{0} \mathbf{I}+\kappa_{1} \mathbf{1}_{z} \mathbf{1}_{z}$, where $\kappa_{0}$ and $\kappa_{1}$ are uniform, which gives the following equation for the mean temperature $\bar{\Theta}$ :

$$
\frac{\partial \bar{\Theta}}{\partial t}+\overline{\mathbf{v}} \cdot \nabla \bar{\Theta}=\left(\kappa_{0} \nabla^{2}+\kappa_{1} \partial_{z z}\right) \bar{\Theta}+\frac{Q}{\rho c_{p}} .
$$

Equation (3) includes advection due to a mean velocity $\overline{\mathbf{v}}$. The solution method for (1) presented herein reduces the heat conduction problem to an equivalent heat problem in a sphere, with anisotropic heat conduction which depends on the ellipticity of the spheroid. The anisotropic conduction has the same form as the turbulent anisotropic thermal diffusion due to rapid rotation in (3). The spheroidal heat conduction problem without advection is considered here since it has an alternative analytic solution in terms of spheroidal wave functions, with which to compare the method. Further motivation came from a study of thermal convection in oblate spheroids [4]. Oblateness is a natural consequence of rotation in planetary and astrophysical objects, although it is typically rather weak. Spheroids also offer the simplest departure from a spherical geometry regardless of their ellipticity.

The spheroidal heat conduction problem with a homogeneous Dirichlet boundary condition (2) is formulated mathematically in Section 2. The method of solution is described in Section 3. In Section 4 the solution in terms of spheroidal wave functions is outlined. The numerical and analytic solutions are compared in Section 5. Section 6 contains concluding remarks.

\section{Formulation of the problem}

Define the boundary of the solid in (dimensional) Cartesian coordinates $\left(x_{*}, y_{*}, z_{*}\right)$ is $r=1$, where $r(r \geq 0)$ is defined by

$$
r^{2}:=\frac{x_{*}^{2}+y_{*}^{2}}{a_{*}^{2}}+\frac{z_{*}^{2}}{b_{*}^{2}} .
$$




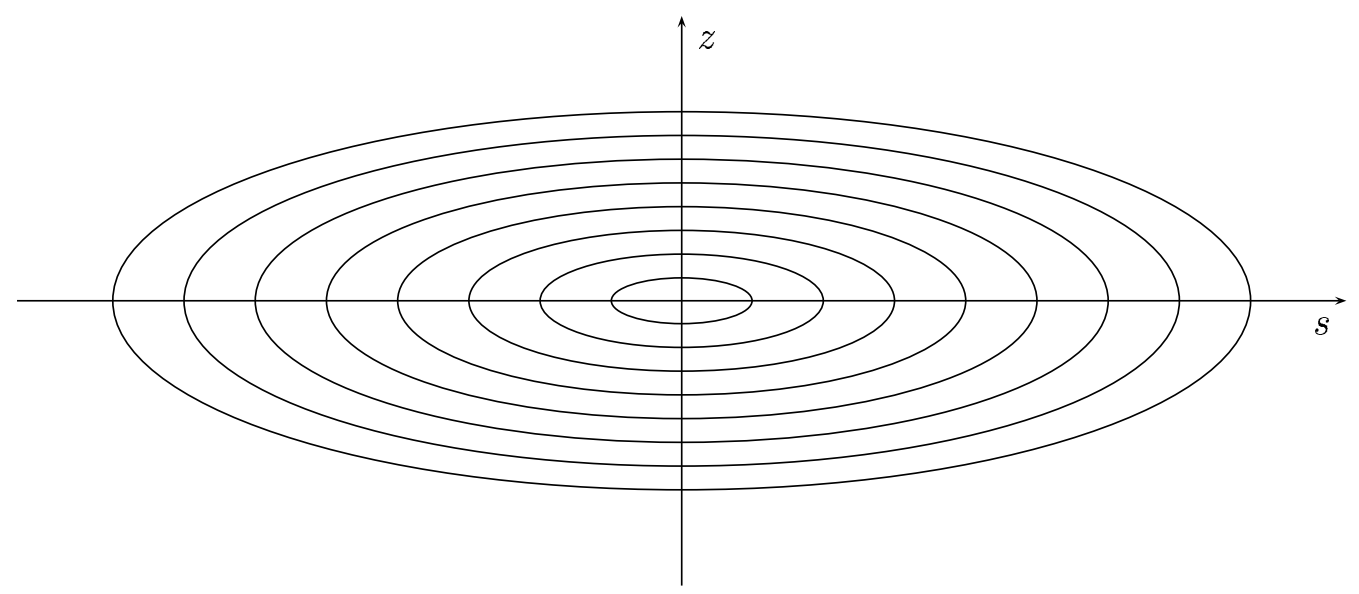

Figure 1: Homeoidal oblate spheroids of identical semi-axis ratio $a / b=3$. The horizontal label $s=\sqrt{x^{2}+y^{2}}$.

The asterisks indicate dimensional quantities. When $a_{*}>b_{*}$, the level surfaces of the variable $r$ are homeoidal oblate spheroids of semi-major axis $r a_{*}$, semi-minor axis $r b_{*}$ and identical ellipticity $e:=\sqrt{1-\left(b_{*} / a_{*}\right)^{2}}$, but their foci are at $s:=\sqrt{x^{2}+y^{2}}=e r a_{*}, z=0$, so they are not confocal. See Figure 1. Note particularly that $r$ is not the spherical radius $\sqrt{x^{2}+y^{2}+z^{2}}$. When $a_{*}<b_{*}$, the $r$-surfaces are homeoidal prolate spheroids of semi-axes $r a_{*}, r b_{*}$, ellipticity $e:=\sqrt{1-\left(a_{*} / b_{*}\right)^{2}}$ and foci $s=0, z= \pm e r b_{*}$. The $z$-axis is the symmetry axis of the spheroids.

The problem is non-dimensionalised using a typical length scale $\mathcal{L}$, the thermal diffusion time scale $\mathcal{L}^{2} / \kappa$ and a heat source $\mathcal{L}^{2} / \rho c_{p} \kappa$. The dimensionless Cartesian coordinates and semi-axes are $x=x_{*} / \mathcal{L}, y=y_{*} / \mathcal{L}, z=z_{*} / \mathcal{L}$, $a=a_{*} / \mathcal{L}$ and $b=b_{*} / \mathcal{L}$. The quantity $r$ is dimensionless and the boundary remains $r=1$. The operator $\nabla:=\mathbf{1}_{x} \partial_{x}+\mathbf{1}_{y} \partial_{y}+\mathbf{1}_{z} \partial_{z}$ is dimensionless. The dimensionless form of the heat equation (1) for the temperature $\Theta$ is thus

$$
\partial_{t} \Theta=\nabla^{2} \Theta+Q
$$


Useful choices for the length scale $\mathcal{L}$ are the semi-axes $a_{*}$ or $b_{*}$, and the equi-volume radius $\left(a_{*}^{2} b_{*}\right)^{1 / 3}$, since the volume is $4 \pi a_{*}^{2} b_{*} / 3$. Herein $\mathcal{L}=b_{*}$ and $\mathcal{L}=a_{*}$ are used in the oblate and prolate cases respectively. On the boundary $r=1$ the temperature vanishes:

$$
\Theta=0 \quad \text { on } \quad r=1
$$

\section{Numerical method of solution}

\subsection{A homeoidal spheroidal coordinate system}

Introduce the angular coordinate $\theta$ and east-longitude $\phi$ in dimensionless form,

$$
x=\operatorname{ar} \sin \theta \cos \phi, \quad y=\operatorname{ar} \sin \theta \sin \phi, \quad z=b r \cos \theta .
$$

Figure 2 shows the coordinates $(r, \theta, \phi)$ of a point $P(x, y, z)$ on the oblate spheroid $(a>b)$ for a fixed value of $r$ in a meridional section of fixed $\phi$. In particular, $\theta$ is the angle $\angle N O Q$, not the co-latitude $\angle N O P$ of $P$, and $a r$ is the radius of the sphere escribed on the spheroid. The coordinate system $(r, \theta, \phi)$ is not orthogonal.

\subsection{Stretched coordinates and equations}

To proceed stretch the Cartesian coordinates $\widetilde{x}=x / a, \widetilde{y}=y / a$ and $\widetilde{z}=z / b$, that is, $\widetilde{\mathbf{r}}=\mathbf{L} \cdot \mathbf{r}$, where $\mathbf{L}$ is the diagonal scale tensor, $\mathbf{L}:=a^{-1}\left(\mathbf{1}_{x} \mathbf{1}_{x}+\right.$ $\left.\mathbf{1}_{y} \mathbf{1}_{y}\right)+b^{-1} \mathbf{1}_{z} \mathbf{1}_{z}$, with inverse, $\mathbf{L}^{-1}:=a\left(\mathbf{1}_{x} \mathbf{1}_{x}+\mathbf{1}_{y} \mathbf{1}_{y}\right)+b \mathbf{1}_{z} \mathbf{1}_{z}$. The stretching transforms the level surfaces of $r$ from spheroids into spheres. The variables $(r, \theta, \phi)$ introduced in $(7)$ are spherical polar coordinates in $(\widetilde{x}, \widetilde{y}, \widetilde{z})$-space. The operator $\widetilde{\nabla}$ is related to $\nabla$ by $\mathbf{L}^{-1} \cdot \nabla=\widetilde{\nabla}$. 


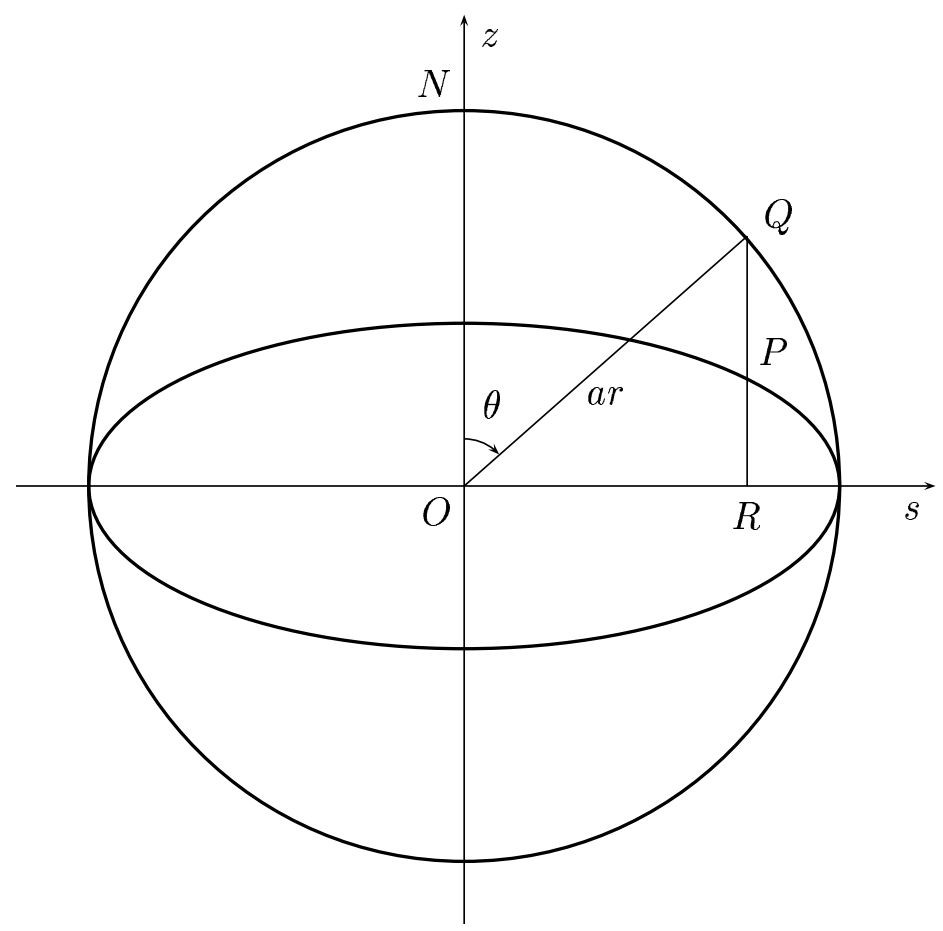

Figure 2: Oblate homeoidal spheroidal coordinates $r, \theta$ of $P$ in the meridional plane through $P$.

Equation (5) becomes

$$
\partial_{t} \Theta=\widetilde{\nabla} \cdot(\mathbf{D} \cdot \widetilde{\nabla} \Theta),
$$

where the diffusion matrix $\mathbf{D}:=\mathbf{L} \cdot \mathbf{L}$ is

$$
\mathbf{D}=\frac{1}{a^{2}}\left(\mathbf{I}+\frac{e^{2}}{1-e^{2}} \mathbf{1}_{z} \mathbf{1}_{z}\right) \quad \text { or } \quad \mathbf{D}=\frac{1}{a^{2}}\left(\mathbf{I}-e^{2} \mathbf{1}_{z} \mathbf{1}_{z}\right)
$$

in the oblate and prolate cases respectively. Expanding $\mathbf{D}$ yields the oblate and prolate equations respectively,

$$
\partial_{t} \Theta=\frac{1}{a^{2}}\left(\widetilde{\nabla}^{2}+\frac{e^{2}}{1-e^{2}} \partial_{\tilde{z} \tilde{z}}\right) \Theta \quad \text { or } \quad \partial_{t} \Theta=\frac{1}{a^{2}}\left(\widetilde{\nabla}^{2}-e^{2} \partial_{\tilde{z} \tilde{z}}\right) \Theta
$$


The features of these equations, which are new over thermal conduction in a sphere, are the anisotropic terms. Thermal conductivity is enhanced (diminished) in the $z$-direction by the anisotropy in the oblate (prolate) case. The boundary condition (6) becomes

$$
\Theta=0 \quad \text { at } r=1 .
$$

\subsection{Spectral equations}

Expand the temperature $\Theta$ in spherical harmonics $Y_{n}^{m}(\theta, \phi)$,

$$
\Theta=\sum_{n, m} \Theta_{n}^{m}(r, t) Y_{n}^{m}(\theta, \phi)
$$

where the spherical harmonic of degree $n$ and order $m$ is

$$
Y_{n}^{m}(\theta, \phi):=(-1)^{m} \sqrt{2 n+1} \sqrt{\frac{(n-m) !}{(n+m) !}} P_{n}^{m}(\cos \theta) e^{i m \phi} .
$$

The associated Legendre function of the first kind $P_{n}^{m}$ is Neumann or Ferrer's form defined by

$$
P_{n, m}(z)=(-1)^{n} \frac{1}{2^{n} n !}\left(1-z^{2}\right)^{m / 2} \frac{d^{n+m}\left(1-z^{2}\right)^{n}}{d z^{n+m}} .
$$

These spherical harmonics are orthonormal with respect to the inner product, $(f, g):=\frac{1}{4 \pi} \oint f g^{*} d \Omega$, and $Y_{n}^{m}=(-1)^{m}\left(Y_{n}^{-m}\right)^{*}$, where the superscript asterisk denotes complex conjugation. $Y_{n}^{m}$ is symmetric (antisymmetric) about the equator if $n-m$ is even (odd),

$$
Y_{n}^{m}(\pi-\theta, \phi)=(-1)^{n-m} Y_{n}^{m}(\theta, \phi) .
$$

Spherical harmonics usefully separate variables in the Laplacian,

$$
\nabla^{2} f(r) Y_{n}^{m}=Y_{n}^{m} D_{n} f, \quad D_{n} f:=\frac{1}{r^{2}} \partial_{r}\left(r^{2} \partial_{r} f\right)-\frac{n(n+1)}{r^{2}} f .
$$


Derive the spectral expansion of $\partial_{\tilde{z} \widetilde{z}} \Theta$ using the following recurrence relations satisfied by the $Y_{n}^{m}$,

$$
\begin{aligned}
& \cos \theta Y_{n}^{m}=c_{n+1}^{m} Y_{n+1}^{m}+c_{n}^{m} Y_{n-1}^{m}, \\
& \sin \theta \partial_{\theta} Y_{n}^{m}=n c_{n+1}^{m} Y_{n+1}^{m}-(n+1) c_{n}^{m} Y_{n-1}^{m},
\end{aligned}
$$

where $c_{n}^{m}:=\sqrt{\left(n^{2}-m^{2}\right) /\left(4 n^{2}-1\right)}$, and the operator identity $\partial_{\tilde{z}}=\cos \theta \partial_{r}-$ $r^{-1} \sin \theta \partial_{\theta}$. Applying these to (12) gives

$$
\partial_{\tilde{z}} \Theta=\sum_{n, m}\left\{c_{n}^{m}\left(\partial_{r}-\frac{n-1}{r}\right) \Theta_{n-1}^{m} c_{n+1}^{m}\left(\partial_{r}+\frac{n+2}{r}\right) \Theta_{n+1}^{m}\right\} Y_{n}^{m} .
$$

One iteration gives the oblate and prolate spectral heat equations respectively,

$$
\partial_{t} \Theta_{n}^{m}=\frac{1}{a^{2}}\left(D_{n} \Theta_{n}^{m}+\frac{e^{2}}{1-e^{2}} \Theta_{\tilde{z} \tilde{z}, n}^{m}\right) \quad \text { or } \quad \partial_{t} \Theta_{n}^{m}=\frac{1}{a^{2}}\left(D_{n} \Theta_{n}^{m}-e^{2} \Theta_{\tilde{z} \tilde{z}, n}^{m}\right)
$$

where

$\Theta_{\widetilde{z} \tilde{z}, n}^{m}=c_{n-1, n}^{m} D_{1-n, 2-n} \Theta_{n-2}^{m}+\left(c_{n, n}^{m}+c_{n+1, n+1}^{m}\right) D_{n} \Theta_{n}^{m}+c_{n+1, n+2}^{m} D_{n+2, n+3} \Theta_{n+2}^{m}$, $c_{n_{1}, n_{2}}^{m}=c_{n_{1}}^{m} c_{n_{2}}^{m}$ and $D_{n_{1}, n_{2}}$ is the second-order radial differential operator, $D_{n_{1}, n_{2}}:=\left(\partial_{r}+n_{1} / r\right)\left(\partial_{r}+n_{2} / r\right)$. The $m=0, n=2$ term contains a nonzero contribution from $\Theta_{0}^{0}$ and hence the $m=0, n=0$ spectral equation must be included. On the boundary the temperature vanishes by (11):

$$
\Theta_{n}^{m}=0 \quad \text { at } r=1 .
$$

By property (14) the spectral equations decouple into two spherical harmonic chains for each $m \geq 0$ : the odd chain, $\Theta_{m+1}^{m}, \Theta_{m+3}^{m}, \ldots$; and the even chain, $\Theta_{m}^{m}, \Theta_{m+2}^{m}, \ldots$ If the spherical harmonic expansion (12) is truncated at $n=N$, then the number of $\Theta_{n}^{m}$ coefficients is $N_{o}:=N-m-\left[\frac{1}{2}(N-m)\right]$, if $n-m$ is odd, and $N_{e}:=\left[\frac{1}{2}(N-m)\right]+1$, if $n-m$ is even, where the brackets indicate the integer part. 
The highest derivative, which occurs in either spectral heat equation (12) or (13), is second-order in $\Theta_{n}^{m}$. Any radial discretisation is permissible. For simplicity the radial functions are discretised using finite-differences on the uniform grid, $r_{j}=j h, j=0: J$, where the grid spacing $h=1 / J$. The spectral heat equation (12) is discretised at the interior points $r_{j}, j=1: J-1$ using the second-order centred finite-difference formulas,

$$
f_{0}^{(1)}=\frac{-f_{-1}+f_{1}}{2 h}-\frac{1}{6} f^{(3)}(\eta) h^{2}, \quad f_{0}^{(2)}=\frac{f_{-1}-2 f_{0}+f_{1}}{h^{2}}-\frac{1}{12} f^{(4)}(\eta) h^{2},
$$

where the subscripts indicate relative position. The number of unknowns and equations is $(J-1) N_{o}$ in the odd problem and $(J-1) N_{e}$ in the even problem.

\section{Spheroidal wave function solutions}

For solutions with separable time-dependence, $\Theta=\widehat{\Theta}(\mathbf{r}) \exp (\gamma t)$, the heat equation (5) with $Q=0$ reduces to an eigenproblem for the growth rate $\gamma$,

$$
\left(\nabla^{2}-\gamma\right) \widehat{\Theta}=0 .
$$

The transformation from Cartesian coordinates to confocal prolate spheroidal coordinates is

$x=\frac{1}{2} d \sqrt{\left(1-\eta^{2}\right)\left(\xi^{2}-1\right)} \cos \phi, \quad y=\frac{d}{2} \sqrt{\left(1-\eta^{2}\right)\left(\xi^{2}-1\right)} \sin \phi, \quad z=\frac{d}{2} \eta \xi$.

with $-1 \leq \eta \leq 1,1 \leq \xi<\infty, 0 \leq \phi \leq 2 \pi$. The boundary (4) with $r=1$ corresponds to $\xi=b / \sqrt{b^{2}-a^{2}}$ and fixes $d=2 \sqrt{b^{2}-a^{2}}$. The transformation to confocal oblate spheroidal coordinates is

$$
x=\frac{d}{2} \sqrt{\left(1-\eta^{2}\right)\left(\xi^{2}+1\right)} \cos \phi, \quad y=\frac{d}{2} \sqrt{\left(1-\eta^{2}\right)\left(\xi^{2}+1\right)} \sin \phi, \quad z=\frac{d}{2} \eta \xi,
$$




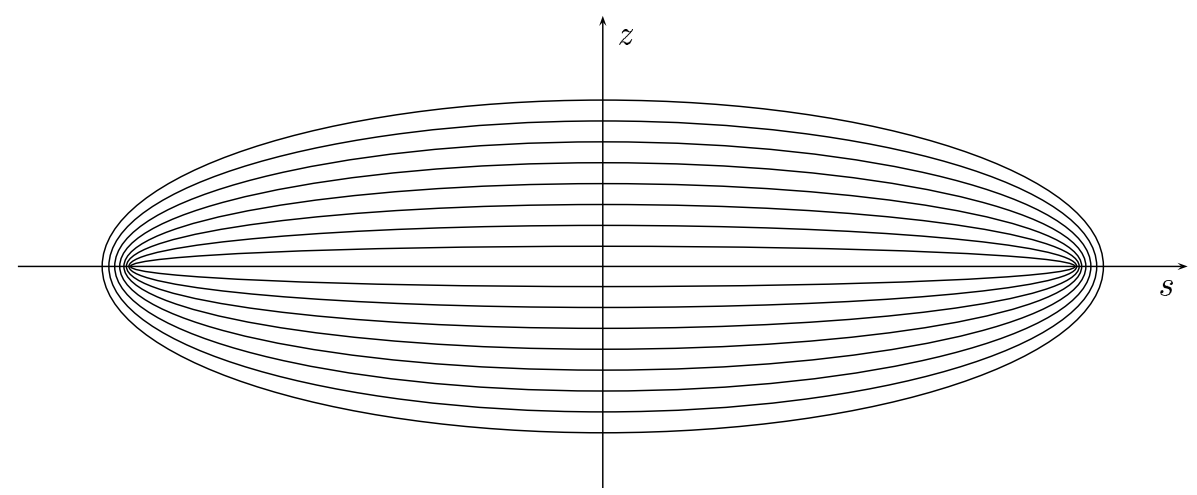

Figure 3: Confocal ellipses of semi-minor axes as in Figure 11. The outermost ellipses in the two figures are identical.

where now $0 \leq \xi<\infty$. In oblate coordinates the boundary $r=1$ corresponds to $\xi=b / \sqrt{a^{2}-b^{2}}$ and $d=2 \sqrt{a^{2}-b^{2}}$. Compare Figures 1 and 3 to see the difference between confocal and homeoidal coordinates.

In confocal prolate spheroidal variables equation (20) becomes

$\left[\frac{\partial}{\partial \eta}\left(1-\eta^{2}\right) \frac{\partial}{\partial \eta}+\frac{\partial}{\partial \xi}\left(\xi^{2}-1\right) \frac{\partial}{\partial \xi}+\frac{\xi^{2}-\eta^{2}}{\left(1-\eta^{2}\right)\left(\xi^{2}-1\right)} \frac{\partial^{2}}{\partial \phi^{2}}+c^{2}\left(\xi^{2}-\eta^{2}\right)\right] \widehat{\Theta}=0$

where the growth rate

$$
\gamma=-\frac{c^{2}}{b^{2}-a^{2}}
$$

The solutions are separable in confocal prolate spheroidal variables,

$$
\widehat{\Theta}=S_{m n}(c, \eta) R_{m n}^{(1)}(c, \xi) e^{i m \phi},
$$

where $n, m$ are integers with $0 \leq m \leq n$ and the degree $n$ and order $m$ prolate spheroidal angular function $S_{m n}(c, \eta)$ and radial function of the first kind $R_{m n}^{(1)}(c, \xi)$ respectively satisfy

$$
\frac{d}{d \eta}\left[\left(1-\eta^{2}\right) \frac{d}{d \eta} S_{m n}(c, \eta)\right]+\left[\lambda_{m n}-c^{2} \eta^{2}-\frac{m^{2}}{1-\eta^{2}}\right] S_{m n}(c, \eta)=0,
$$




$$
\frac{d}{d \xi}\left[\left(\xi^{2}-1\right) \frac{d}{d \xi} R_{m n}^{(1)}(c, \xi)\right]-\left[\lambda_{m n}-c^{2} \xi^{2}+\frac{m^{2}}{\xi^{2}-1}\right] R_{m n}^{(1)}(c, \xi)=0 .
$$

Expand the prolate spheroidal angular function $S_{m n}(c, \eta)$ in a series of associated Legendre functions of the form,

$$
S_{m n}(c, \eta)=\sum_{r=0,1}^{\infty} d_{r}^{m n}(c) P_{m+r}^{m}(\eta),
$$

where the prime on the summation sign indicates summation over even values of $r$ when $n-m$ is even and odd values of $r$ when $n-m$ is odd.

A recurrence relation for the $d_{r}^{m n}$ coefficients can be found [2] by substituting (26) into (25) and using properties of the Legendre differential equation produces a tridiagonal eigenvalue problem for the eigenvalue $\lambda_{m n}(c)$ and the $d_{r}^{m n}(c)$,

$$
\begin{aligned}
& \frac{(2 m+r+2)(2 m+r+1) c^{2}}{(2 m+2 r+3)(2 m+2 r+5)} d_{r+2}^{m n}(c) \\
+ & {\left[(m+r)(m+r+1)-\lambda_{m n}(c)+\frac{2(m+r)(m+r+1)-2 m^{2}-1}{(2 m+2 r-1)(2 m+2 r+3)} c^{2}\right] d_{r}^{m n}(c) } \\
& +\frac{r(r-1) c^{2}}{(2 m+2 r-3)(2 m+2 r-1)} d_{r-2}^{m n}(c)=0, \quad(r \geq 0)
\end{aligned}
$$

Using the expressions

$$
\begin{aligned}
\gamma_{r}^{m} & =(m+r)(m+r+1)+\frac{1}{2} c^{2}\left[1-\frac{4 m^{2}-1}{(2 m+2 r-1)(2 m+2 r+3)}\right], \quad r \geq 0, \\
\beta_{r}^{m} & =\frac{r(r-1)(2 m+r)(2 m+r-1) c^{4}}{(2 m+2 r-1)^{2}(2 m+2 r-3)(2 m+2 r+1)}, \quad r \geq 2, \\
N_{r}^{m} & =\frac{(2 m+r)(2 m+r-1) c^{2}}{(2 m+2 r-1)(2 m+2 r+1)} \frac{d_{r}^{m n}}{d_{r-2}^{m n}}, \quad r \geq 2
\end{aligned}
$$


equation (27) simplifies to a forward or backward two-term recurrence relation

$$
N_{r+2}^{m}=\lambda_{m n}-\gamma_{r}^{m}-\frac{\beta_{r}^{m}}{N_{r}^{m}}, \quad N_{r}^{m}=\frac{\beta_{r}^{m}}{\lambda_{m n}-\gamma_{r}^{m}-N_{r+2}^{m}},
$$

with $N_{2}^{m}=\lambda_{m n}-\gamma_{0}^{m}$ and $N_{3}^{m}=\lambda_{m n}-\gamma_{1}^{m}$. The forward form is used for $r \geq n-m$, the backward form for $0 \leq r \leq n-m$, and the two matched at $r=n-m .{ }^{1}$ The arbitrariness of the coefficients $d_{r}^{m n}(c)$ is removed by normalizing the spheroidal angular function so that it reduces to the corresponding associated Legendre function or its derivative when $c=0$. Thus $S_{m n}(c, 0)=P_{n}^{m}(0)$ for $n-m$ even and $S_{m n}^{\prime}(c, 0)=P_{n}^{m \prime}(0)$ for $n-m$ odd, see [2].

Expand the spheroidal radial function of the first kind in a series of spherical Bessel functions and the same $d_{r}^{m n}(c)$ coefficients previously calculated:

$$
R_{m n}^{(1)}(c, \xi)=\frac{\left(1-\xi^{-2}\right)^{m / 2}}{\sum_{r=0,1}^{\infty} d_{r}^{m n}(c) \frac{(2 m+r) !}{r !}} \sum_{r=0,1}^{\infty}(-1)^{\frac{r+m-n}{2}} d_{r}^{m n}(c) \frac{(2 m+r) !}{r !} j_{m+r}(c \xi)
$$

The Dirichlet boundary condition (6) becomes $R_{m n}^{(1)}\left(c, b / \sqrt{b^{2}-a^{2}}\right)=0$, which determines the parameter $c$ and hence by (24) the growth rate $\gamma$. There is a countably infinite set of $c$ values. Figure 4 shows the first three prolate radial functions and the angular function for $m=1, n=1$ and $b / a=2$.

Separable solutions in confocal oblate spheroidal coordinates are

$$
\Theta=S_{m n}(-i c, \eta) R_{m n}^{(1)}(-i c, i \xi) e^{i m \phi+\gamma t}, \quad \gamma=-\frac{c^{2}}{a^{2}-b^{2}},
$$

where the boundary condition $R_{m n}^{(1)}\left(-i c, i b / \sqrt{a^{2}-b^{2}}\right)=0$ determines the parameter $c$. Figure 5 shows the first three oblate radial functions and the

${ }^{1}$ Note the differences with equations (3.1.7) and (3.1.8) of Flammer [2]. 

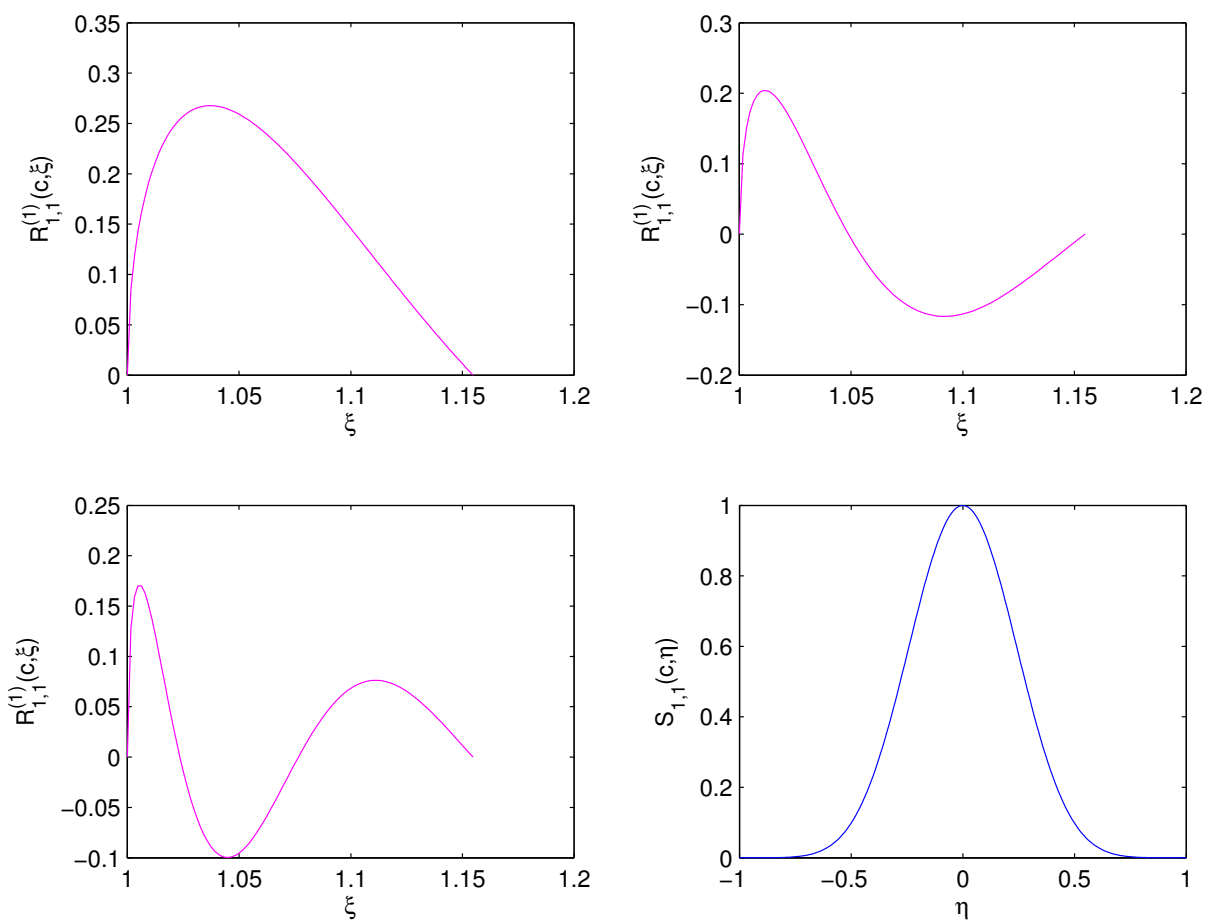

FiguRE 4: The first three prolate radial functions $(c \approx 7.12,12.62,18.09)$ and the angular function for $m=1, n=1$ and $b / a=2$.

angular function for $m=1, n=1$ and $a / b=2$. The poor normalisation of the associated Legendre functions is apparent in the large amplitude of the angular function.

\section{$5 \quad$ Results}

For either method the solutions are modes proportional to $\exp (\gamma t+i m \phi)$ and decouple for different azimuthal parameters $m$. The growth rate $\gamma$ in 

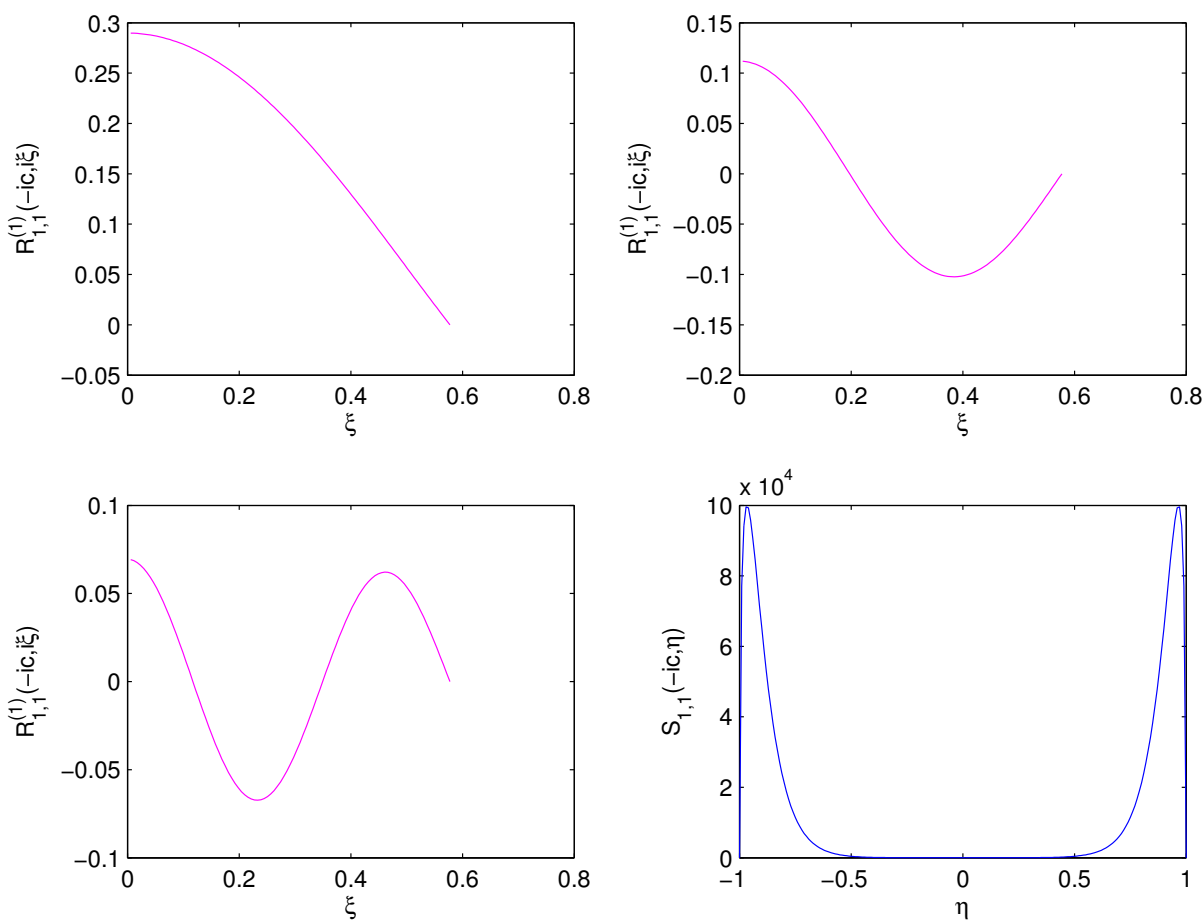

FiguRE 5: The first three oblate radial functions $(c \approx 4.86,10.07,15.47)$ and the angular function for $m=1, n=1$ and $a / b=2$.

the coordinate scaling method is given by the solution of the matrix eigenproblem, $\mathbf{A x}=\gamma \mathbf{x}$, where the matrix $\mathbf{A}$, which arises from the discretisation, is block-tridiagonal in $n$. The $r$-blocks depend on the radial discretisation. Herein they are tridiagonal. The matrix eigenproblem is solved using inverse iteration.

Growth rates calculated for representative parameter values using the scaling method with finite-difference are compared to the spheroidal wave function solutions in Tables 1 and 2. Table 1 shows the level of agreement in the oblate case with azimuthal wavenumber $m=1$ for radial truncation 
TABLE 1: Growth rates of the smallest-c modes for the oblate case with $m=1$.

\begin{tabular}{|r|c|c|c|}
\hline$a / b$ & $n$ & $\gamma$ (finite-difference) & $\gamma$ (wave-function) \\
\hline 2 & 1 & -7.8764156 & -7.8764188 \\
2 & 2 & -18.241998 & -18.242011 \\
3 & 1 & -5.4399222 & -5.4399255 \\
3 & 2 & -14.887684 & -14.887689 \\
4 & 1 & -4.4962828 & -4.4962840 \\
4 & 2 & -13.451924 & -13.451923 \\
\hline
\end{tabular}

TABLE 2: Growth rates of the smallest-c modes for the prolate case with $m=1$.

\begin{tabular}{|r|c|c|c|}
\hline$b / a$ & $n$ & $\gamma$ (finite-difference) & $\gamma$ (wave-function) \\
\hline 2 & 1 & -16.895242 & -16.895258 \\
2 & 2 & -21.763262 & -21.763279 \\
3 & 1 & -16.073760 & -16.073774 \\
3 & 2 & -19.041299 & -19.041309 \\
4 & 1 & -15.699665 & -15.699678 \\
4 & 2 & -17.835484 & -17.835489 \\
\hline
\end{tabular}

$J=1000$ and angular truncation $N=20$. Table 2 shows the level of agreement in the prolate case with $m=1$ for radial truncation $J=1000$ and angular truncation $N=40$.

\section{Concluding remarks}

The accuracy of the results is restricted by the $\mathcal{O}\left(h^{2}\right)$ radial finite differences but the correctness of the method is clear. The method has the advantage of simplicity and readily extends to more complicated spheroidal problems with axisymmetric advection and sources, and inhomogeneous boundary con- 
ditions. Recurrence relations (16) and expansion (17) may be used to derive the additional spectral terms. In fact, (15) and (17) may be used to derive spectral equations for differential operators of the form $P\left(\nabla^{2}, \partial_{\tilde{z}}\right)$, where $P$ is a multinomial in two variables. The spectral equations may also be used in steady state and time stepping problems. Nonlinear and vector problems in spheroidal geometries and ellipsoidal geometries generally are more difficult to implement [4]. Figures 1 and 3 show that homeoidal and confocal spheroidal coordinates lead to quite different spheroidal shells, so results of the method and spheroidal wave functions cannot be compared in that case.

\section{References}

[1] S. I. Braginsky and V. P. Meytlis. Local turbulence in the Earth's core. Geophys. Astrophys. Fluid Dynam., 55:71-87, 1990. C855

[2] C. Flammer. Spheroidal Wave Functions, Stanford University Press, Stanford, California. 1957 C864, C865

[3] D. J. Ivers. Geodynamo models with anisotropic turbulent thermal diffusion. Abstracts of the 9th SEDI (Studies of the Earth's Deep Interior) Symposium, page 41 Garmisch-Partenkirchen, Germany. 2004 C855

[4] D. J. Ivers. Thermal instability of an oblate spheroid. Abstracts of the 8th SEDI (Studies of the Earth's Deep Interior) Symposium, Granlibakken, USA. 2002 C856, C869

[5] C. Niven. On the conduction of heat in ellipsoids of revolution. Phil. Trans. Roy. Soc. Lond., 171:117-151, 1880. C855

[6] G. C. Phillips, and D. J. Ivers. Spherical anisotropic diffusion models for the Earth's core. Phys. Earth Planet. Int., 117:209-223, 2000. C855 
[7] G. C. Phillips and D. J. Ivers. Spectral interactions of rapidly-rotating anisotropic turbulent viscous and thermal diffusion in the Earth's core. Phys. Earth Planet. Int., 128:93-107, 2001. C855

[8] G. C. Phillips and D. J. Ivers. Strong field anisotropic diffusion models for the Earth's core. Phys. Earth Planet. Int., 140:13-28, 2003. C855 